\title{
Characterization of phylogenetic networks with NetTest
}

\author{
Miguel Arenas*1, Mateus Patricio', David Posada' and Gabriel Valiente ${ }^{2}$
}

\begin{abstract}
Background: Typical evolutionary events like recombination, hybridization or gene transfer make necessary the use of phylogenetic networks to properly depict the evolution of DNA and protein sequences. Although several theoretical classes have been proposed to characterize these networks, they make stringent assumptions that will likely not be met by the evolutionary process. We have recently shown that the complexity of simulated networks is a function of the population recombination rate, and that at moderate and large recombination rates the resulting networks cannot be categorized. However, we do not know whether these results extend to networks estimated from real data.

Results: We introduce a web server for the categorization of explicit phylogenetic networks, including the most relevant theoretical classes developed so far. Using this tool, we analyzed statistical parsimony phylogenetic networks estimated from 5,000 DNA alignments, obtained from the NCBI PopSet and Polymorphix databases. The level of characterization was correlated to nucleotide diversity, and a high proportion of the networks derived from these data sets could be formally characterized.

Conclusions: We have developed a public web server, NetTest (freely available from the software section at http:// darwin.uvigo.es), to formally characterize the complexity of phylogenetic networks. Using NetTest we found that most statistical parsimony networks estimated with the program TCS could be assigned to a known network class. The level of network characterization was correlated to nucleotide diversity and dependent upon the intra/interspecific levels, although no significant differences were detected among genes. More research on the properties of phylogenetic networks is clearly needed.
\end{abstract}

\section{Background}

The evolution of DNA or protein sequences that have been the subject of reticulating events like recombination, gene transfer, or hybridization cannot be depicted with a single phylogenetic tree [1-3]. On the contrary, phylogenetic networks allow reticulations among branches rather than imposing a strictly bifurcating structure, and therefore are much more suitable for this task [4-6]. There are different types of phylogenetic networks [5]. Among these, split networks (implicit representation) are obtained as a combinatorial generalization of phylogenetic trees, and are designed to represent incompatibilities within and between data sets. In order

\footnotetext{
*Correspondence: miguelab@uvigo.es

1 Department of Biochemistry, Genetics and Immunology, University of Vigo, E-36310 Vigo, Spain

Full list of author information is available at the end of the article
}

to be able to accommodate incompatible splits, they contain internal nodes that do not represent ancestral genes or sequences. On the other hand, reticulate networks (explicit representation) represent evolutionary histories in the presence of reticulate events such as hybridization, horizontal gene transfer, or recombination, and every internal node represents an inferred ancestor. Most often, phylogenetic networks estimated from real data are depicted as unrooted, especially at the intraspecific level.

Research on phylogenetic networks is just starting, especially if we compare it with all the work that has been done on bifurcating trees, which, indeed, are much simpler structures. This means that many of the calculations that can be easily implemented for bifurcating trees are not available for phylogenetic networks. For example, typically researchers are interested in contrasting different biological hypotheses through the comparison of 
alternative evolutionary histories. In the absence of reticulation a variety of appropriate metrics exist to measure the distance between two phylogenetic trees (see [7-10]), but in the case of networks this issue is much more complicated.

"Perfect" -that is, that obey non-negativity, separation, symmetry, and triangle inequality-comparison metrics for networks have already been proposed [11-18], together with recognition algorithms that allow for the classification of networks into specific categories. Indeed, the formal characterization of phylogenetic networks is important if we want to compare reticulate hypotheses within a sound framework. For example, a web server http://dmi.uib.es/ gcardona/BioInfo/alignment.php was recently developed by G. Cardona to compare and align explicit phylogenetic networks based on the $\mu$-distance metric [19]. The problem is that from the evolutionary point of view, these categories imply stringent assumptions. We have shown that population genetic models like the coalescent with recombination can result into uncharacterizable phylogenetic networks [20]. However, it is unknown whether this is also the case for networks estimated from real data.

\section{Definitions}

A network contains nodes (vertices) and branches (edges) connecting them. Here we will refer to rooted networks (rooted directed acyclic graphs) with a temporal reference that allows for the identification of parent and child (descendant) nodes. Internal nodes have one or two children while external nodes (leaves) have none. The oldest node is called the root and has no parents. Tree nodes and hybrid nodes have one and two parents, respectively. Internal tree nodes have two children, while hybrid nodes have only one child. Nodes that share the same parent are siblings. Networks can be classified as tree sibling [12,13], where every hybrid node has at least one sibling that is a tree node; tree child $[11,13,14]$, in which every internal node has at least one child that is a tree node; galled-trees $[15,16,21]$, where the paths from the most recent common ancestor (MRCA) of the parents of a hybrid node down to the hybrid node form disjoint cycles; and (binary) trees, which only contain tree nodes. These network classes are nested: tree-sibling ? tree-child ? galledtrees ? trees, meaning a tree is also a galled-tree, a treechild network, and a tree-sibling network, and so on. Networks that cannot be included in any of these categories are considered uncharacterizable. There is also a nested classification of networks as level-k networks [22], where a level-0 network is a tree, a level-1 network is a galled-tree, and, in general, a network is a level-k network if every biconnected component has at most $k$ hybrid nodes. Perfect metrics have also been proposed for levelk networks [23].

\section{Implementation}

\section{Implementation of the NetTest server}

The NetTest web server is composed of an HTML frontpage in which the user can upload or paste the network in different formats (see Results). The analysis pipeline was developed in Perl using CGI (Common Gateway Interface). The network classification uses the BioPerl module Bio::PhyloNetwork [13]. The web server uses the Apache HTTP server and was tested and verified using Firefox, Safari, and Internet Explorer.

\section{Characterization of Reticulate Networks from Empirical Data}

We used NetTest to analyze a large group of nucleotide alignments from the PopSet database at the NCBI http:// www.ncbi.nlm.nih.gov/sites/entrez?db=popset (Figure 1). We downloaded all available data sets for four genes: POL (viral polymerase), COX1 (mitochondrial cytochrome oxidase I), $18 \mathrm{~S}$ ribosomal RNA (small eukaryotic ribosomal subunit), and ITS1 (ribosomal internal transcribed spacer). In total, we analyzed 565, 1407, 2294, and 191 data sets, respectively, each containing between 9 and 50 sequences. All data sets were aligned using MAFFT [24].

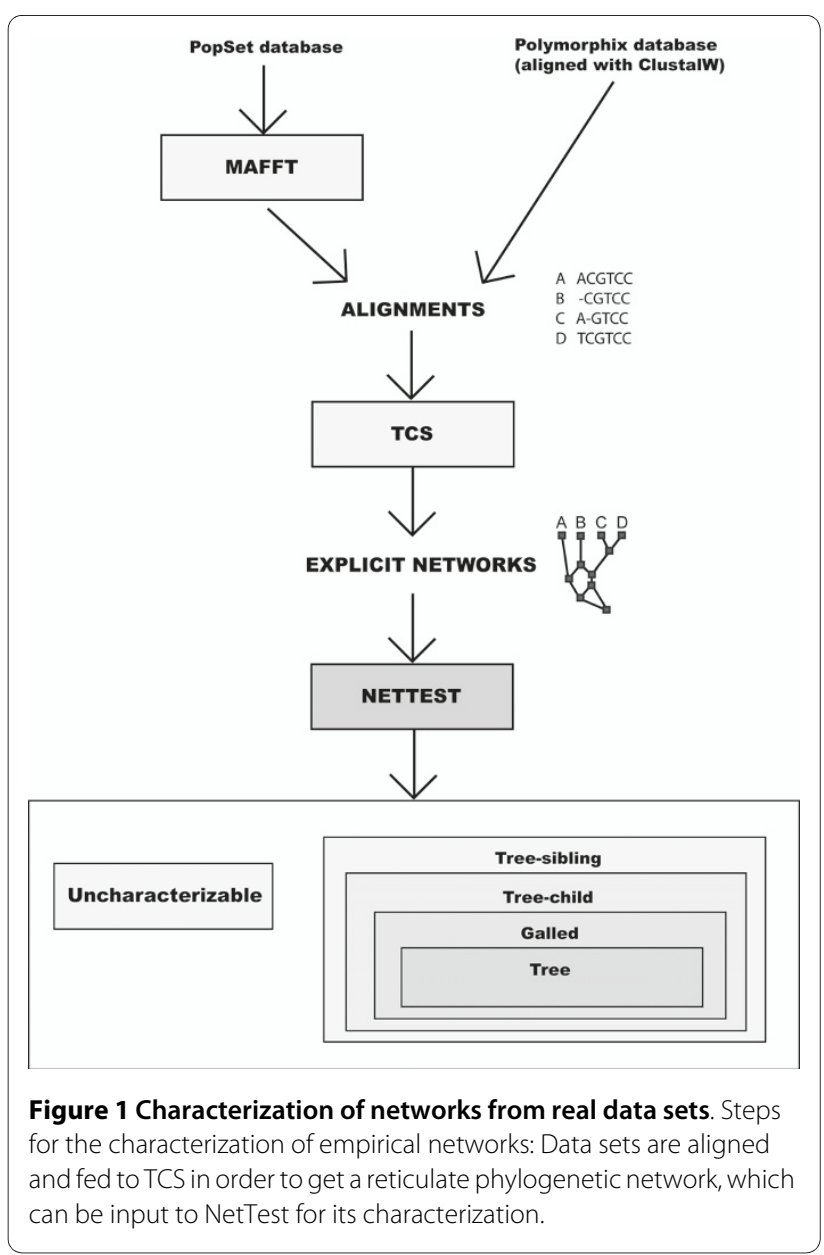


Table 1: Characterization of TCS networks.

\begin{tabular}{|c|c|c|c|c|c|}
\hline Nature of data sets & POL & Cox1 & $18 S$ & ITS1 & Human \\
\hline Number of data sets & 566 & 1417 & 2303 & 191 & 516 \\
\hline $\begin{array}{l}\text { Number of } \\
\text { networks generated } \\
\text { by TCS }\end{array}$ & 1535 & 3889 & 6041 & 531 & 1035 \\
\hline Hybrid nodes & 4950.32 & 8910.23 & 9140.15 & 1170.22 & 1240.12 \\
\hline Tree & $\begin{array}{c}1326 \\
(86.4 \%) \\
{[00.00]}\end{array}$ & $\begin{array}{c}3428 \\
(88.1 \%) \\
{[00.00]}\end{array}$ & $\begin{array}{c}5505 \\
(91.1 \%) \\
{[00.00]}\end{array}$ & $\begin{array}{c}465 \\
(87.6 \%) \\
{[00.00]}\end{array}$ & $\begin{array}{c}952 \\
(92.0 \%) \\
{[00.00]}\end{array}$ \\
\hline Galled-tree & $\begin{array}{c}1443 \\
(117) \\
(94.0 \%) \\
{[1311.11]}\end{array}$ & $\begin{array}{c}3738 \\
(310) \\
(96.1 \%) \\
{[3481.12]}\end{array}$ & $\begin{array}{c}5873 \\
(368) \\
(97.2 \%) \\
{[4101.11]}\end{array}$ & $\begin{array}{c}507 \\
(42) \\
(95.5 \%) \\
{[531.26]}\end{array}$ & $\begin{array}{c}1015 \\
(63) \\
(98.1 \%) \\
{[671.06]}\end{array}$ \\
\hline Tree-child & $\begin{array}{c}1495 \\
(52) \\
(97.4 \%) \\
{[1613.10]}\end{array}$ & $\begin{array}{c}3799 \\
(61) \\
(97.7 \%) \\
{[1692.77]}\end{array}$ & $\begin{array}{c}5941 \\
(68) \\
(98.3 \%) \\
{[1792.63]}\end{array}$ & $\begin{array}{c}518 \\
(11) \\
(97.6 \%) \\
{[272.45]}\end{array}$ & $\begin{array}{c}1022 \\
(7) \\
(98.7 \%) \\
{[162.29]}\end{array}$ \\
\hline Tree-sibling & $\begin{array}{c}1523 \\
(28) \\
(99.2 \%) \\
{[1033.68]}\end{array}$ & $\begin{array}{c}3865 \\
(66) \\
(99.4 \%) \\
{[2413.65]}\end{array}$ & $\begin{array}{c}6019 \\
(78) \\
(99.6 \%) \\
{[2322.97]}\end{array}$ & $\begin{array}{c}523 \\
(5) \\
(98.5 \%) \\
{[112.20]}\end{array}$ & $\begin{array}{c}1033 \\
(11) \\
(99.8 \%) \\
{[363.27]}\end{array}$ \\
\hline Uncharacterizable & $\begin{array}{c}12 \\
(0.8 \%) \\
{[1008.33]}\end{array}$ & $\begin{array}{c}24 \\
(0.6 \%) \\
{[1335.54]}\end{array}$ & $\begin{array}{c}22 \\
(0.4 \%) \\
{[934.23]}\end{array}$ & $\begin{array}{c}8 \\
(1.5 \%) \\
{[263.25]}\end{array}$ & $\begin{array}{c}2 \\
(0.2 \%) \\
{[52.50]}\end{array}$ \\
\hline
\end{tabular}

For each gene, the table indicates the total number of data sets studied (both databases), the total number of networks generated by TCS, the total number and mean (per network) of reticulate nodes and the number of networks classified as tree, galled-tree, tree-child, and treesibling. In parenthesis we indicate the number of networks in that category that did not belong to the previous category and the percentage of characterized networks. In brackets we indicated the number and mean (per network) of reticulate nodes. The last row indicates the number of uncharacterizable networks (i.e., more complex than tree-sibling).

In addition, we also gathered 516 alignments from the Polymorphix database [25], representing a variety of nuclear genes from Homo sapiens. This database uses ClustalW [26] to generate the alignments.

All the alignments were fed into the TCS program [27] for the estimation of statistical parsimony networks [28] under the default $95 \%$ connectivity limit, which is the maximum number of mutational connections between pairs of sequences justified by the "parsimony" criterion [28]. Note that the presence of divergent sequences can result in the generation of several TCS (sub)networks, in which case were analyzed independently. In a few cases $(<1 \%)$ the program crashed before completing the analysis, and the corresponding data sets were excluded from the analyses. The resulting networks were rooted accord- ing to the node with the highest outgroup weight (i.e., the rooting probabilities described in [29]).

\section{Results}

\section{NetTest: A web server for the characterization of explicit} networks

We have developed a web server called NetTest, available from the software section at http://darwin.uvigo.es, able to classify phylogenetic networks into several formal categories (tree, galled-tree, tree-child, and tree-sibling). As far as we know, no other similar programs or servers implement this analysis. All the submitted networks have to be rooted (directed), but they can be specified in different formats: 


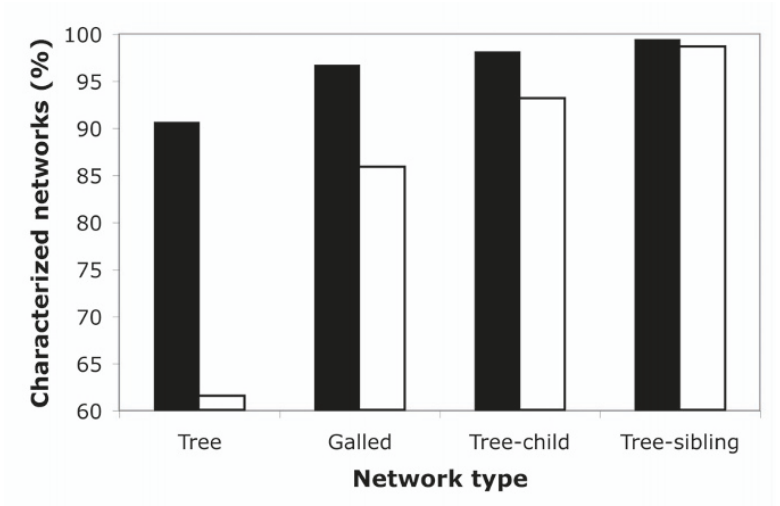

Figure 2 Network complexity and intra/interspecific level. Characterization of TCS networks derived from the PopSet data sets according to their intraspecific (black bars) and interspecific (white bars) composition.

- Directed GML: The Graph Modelling Language is the standard file format in the Graphlet graph editor system (see http://www.infosun.fim.uni-passau.de/ Graphlet/GML/).

- TCS-GML: The program TCS [27] can generate unrooted networks in GML format (saved as *.graph files). In this case, the network is automatically directed by NetTest assuming that the "root" is the node with the highest outgroup weight. If the file submitted to the server contains several networks, NetTest analyzes them in a sequential fashion.

- NEXUS-SplitsTree: The program SplitsTree [30] can produce rooted networks using the "rooted equal angle algorithm" that can be exported in NEXUS format (".nex) and feeded directly to NetTest.

- Extended Newick (eNewick) [19]: This is the type of representation used for the directed networks simulated with Netgen [31] or estimated with Phylonet

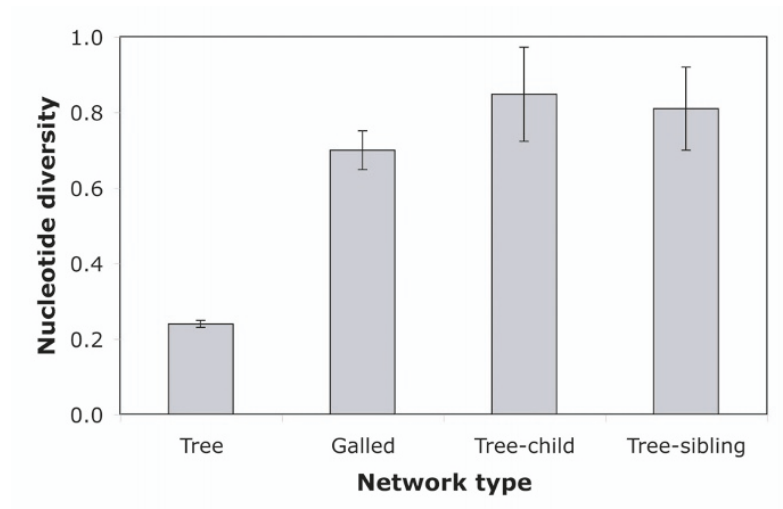

Figure 3 Network complexity and diversity. Characterization of TCS networks derived from the PopSet data sets according to nucleotide diversity. Error bars indicate 95\% confidence intervals.
[32]. The leftmost occurrence of each hybrid node in an eNewick string corresponds to the full description of the subnetwork rooted at that node.

- DIMACS standard [33]: This is a widely used format for representing graphs, developed for the DIMACS Challenge http://dimacs.rutgers.edu/Challenges/. NetTest will consider that all edges are directed and that the first node of each edge is the parent node.

- Branch list: A simple list of connections node $x$ to node $y$, where $x$ corresponds to the parent node and $y$ to the descendant node.

The main output of NetTest is a depiction of the network and an indication of whether it can be characterized under the tree, galled-tree, tree-child, and tree-sibling categories. The results of the analysis can also be downloaded. NetTest also implements a feedback for questions, requests, and bug reports, and a help page is included with detailed information.

\section{Network classes resulting from empirical data}

Using NetTest we found that most networks (98.5-99.6\%) could be characterized regardless of the gene analyzed (Table 1). The analysis of the human data sets also resulted in a high number of characterizable networks (Table 1). For PopSet data sets, those networks containing a majority of sequences corresponding to the same species (intraspecific data sets) resulted in a significantly higher percentage ( $\chi^{2} P$-value $\left.<2.2 \mathrm{e}^{-16}\right)$ of characterization for any class assignment (especially for tree, galledtree, and tree-child) than networks derived from data sets where several sequences belonged to more than one species (interspecific data sets) (see also Figure 2). Also, the level of characterization depended on nucleotide diversity (ANOVA $P$-value $<2 \times 10^{-16}$ ). In particular, data sets resulting in trees were significantly less diverse than data sets resulting in more complex networks (Figure 3).

\section{Discussion and Conclusions}

NetTest is able to analyze rooted phylogenetic networks in several formats and assign them to different network classes -those for which perfect metrics exist. The application is useful not only for theoretical studies, for example in the design of new metrics, but also for measuring the complexity of the evolutionary processes in real or simulated data sets.

Using NetTest we have shown that most of the TCS networks resulting from the analysis of real data can be classified as tree-sibling, tree-child, or galled-tree networks. Indeed, this result is dependent on the fact that TCS was designed for the analysis of closely related sequences. The TCS connection limit forces complex networks to break apart into simpler ones, therefore favouring their assignment to known network categories. This also explains why the TCS networks are simpler than 
the ancestral recombination graphs simulated with the coalescent [20].

Appropriate metrics exist for the comparison of empirical characterizable networks (see for example [13]) like those produced by TCS, allowing us to contrast reticulate hypotheses in a formal fashion. Only a small fraction of the TCS networks estimated here were more convoluted than any of these classes, suggesting that more research is needed if we want to compare and analyze more complex phylogenetic networks estimated from real data.

\section{Availability and requirements}

\section{Project name: NetTest}

Project home: http://darwin.uvigo.es, software section

Operating system(s): Platform independent

Programming language: Perl with BioPerl library

Requirements: None

Licence: GNU GPL

Any restrictions to use by non-academics: None

\section{Authors' contributions}

MA and GV carried out the network analyses. MP and MA gathered the data sets and MP developed the web server. DP conceived and coordinated the study. All authors participated in its design and helped to draft the manuscript. All authors read and approved the final manuscript.

\section{Acknowledgements}

M.A. was supported by an FPI fellowship BES-2005-9151 (Spanish Ministry of Education and Science [MEC]). D.P. was partially supported by MEC grant BIO2007-61411. G.V. was partially supported by the Spanish government and the EU FEDER program under project MTM2006-07773 COMGRIO.

\section{Author Details}

'Department of Biochemistry, Genetics and Immunology, University of Vigo, E36310 Vigo, Spain and ${ }^{2}$ Algorithms, Bioinformatics, Complexity and Formal Methods Research Group, Technical University of Catalonia, E-08034 Barcelona, Spain

Received: 9 March 2010 Accepted: 20 May 2010

Published: 20 May 2010

\section{References}

1. Posada D, Crandall KA: The effect of recombination on the accuracy of phylogeny estimation. J Mol Evol 2002, 54(3):396-402.

2. Schierup $\mathrm{MH}$, Hein J: Consequences of recombination on traditional phylogenetic analysis. Genetics 2000, 156:879-891.

3. Schierup MH, Mikkelsen AM, Hein J: Recombination, balancing selection and phylogenies in MHC and self-incompatibility genes. Genetics 2001, 159(4):1833-1844.

4. Posada D, Crandall KA: Intraspecific gene genealogies: trees grafting into networks. Trends in Ecology and Evolution 2001, 16(1):37-45.

5. Huson DH, Bryant D: Application of phylogenetic networks in evolutionary studies. Mol Biol Evol 2006, 23(2):254-267.

6. Morrison DA: Phylogenetic networks in systematic biology (and elsewhere). In Research Advances in Systematic Biology Edited by: Mohan RM. Trivandrum, India: Global Research Network; 2010:1-48.

7. Robinson DF, Foulds LR: Comparison of phylogenetic trees. Math Biosci 1981, 53:131-147.

8. Kuhner MK, Felsenstein J: A simulation comparison of phylogeny algorithms under equal and unequal evolutionary rates. Mol Biol Evol 1994, 11(3):459-468

9. Soria-Carrasco V, Talavera G, Igea J, Castresana J: The K tree score: quantification of differences in the relative branch length and topology of phylogenetic trees. Bioinformatics 2007, 23(21):2954-2956
10. Alberich R, Cardona G, Roselló F, Valiente G: An algebraic metric for phylogenetic trees. App/ Math Lett 2009, 22(9):1320-1324.

11. Cardona G, Llabrés M, Rosselló F, Valiente G: Metrics for phylogenetic networks I: Generalizations of the Robinson-Foulds metric. IEEE/ACM Trans Comput Biol Bioinform 2009, 6(1):46-61.

12. Cardona G, Llabrés $M$, Rosselló $F$, Valiente $G$ : A distance metric for a class of tree-sibling phylogenetic networks. Bioinformatics 2008, 24(13): 1481-1488

13. Cardona $\mathrm{G}$, Rosselló F, Valiente $\mathrm{G}$ : A perl package and an alignment tool for phylogenetic networks. BMC Bioinformatics 2008, 9:175.

14. Cardona G, Rosselló F, Valiente G: Tripartitions do not always discriminate phylogenetic networks. Math Biosci 2008, 211(2):356-370

15. Gusfield D, Eddhu S, Langley C: The Fine Structure of Galls in Phylogenetic Networks. INFORMS J Comput 2004, 16(4):459-469.

16. Roselló F, Valiente G: All that Glisters is not Galled. Math Biosci 2009, 221:54-59.

17. Cardona G, Llabres M, Rossello F, Valiente G: On Nakhleh's metric for reduced phylogenetic networks. IEEE/ACM Trans Comput Biol Bioinform 2009, 6(4):629-638.

18. Nakhleh L: A Metric on the Space of Reduced Phylogenetic Networks. IEEE/ACM Transactions on Computational Biology and Bioinformatics 2009.

19. Cardona G, Rosselló $F$, Valiente G: Extended Newick: It is time for a standard representation of phylogenetic networks. BMC Bioinformatics 2008, 9:532.

20. Arenas M, Valiente G, Posada D: Characterization of reticulate networks based on the coalescent with recombination. Mol Biol Evol 2008, 25(12):2517-2520

21. Gusfield D, Eddhu S, Langley C: Optimal, efficient reconstruction of phylogenetic networks with constrained recombination. J Bioinform Comput Biol 2004, 2(1):173-213.

22. Choy C, Jansson J, Sadakane K, Sung W: Computing the maximum agreement of phylogenetic networks. Theoretical Computer Science 2005, 335(1):93-107.

23. Asano T, Jansson J, Sadakane K, Uehara R, Valiente G: Faster Computation of the Robinson-Foulds Distance between Phylogenetic Networks. In Proc 21st Ann Symp Volume 6129. Combinatorial Pattern Matching. Lecture Notes in Computer Science; 2010:190-201.

24. Katoh K, Toh H: Recent developments in the MAFFT multiple sequence alignment program. Brief Bioinform 2008, 9(4):286-298.

25. Bazin E, Duret L, Penel S, Galtier N: Polymorphix: a sequence polymorphism database. Nucleic Acids Res 2005:D481-484.

26. Thompson JD, Higgins DG, Gibson TJ: CLUSTAL W: improving the sensitivity of progressive multiple sequence alignment through sequence weighting, position-specific gap penalties and weight matrix choice. Nucleic Acids Res 1994, 22(22):4673-4680.

27. Clement M, Posada D, Crandall KA: TCS: a computer program to estimate gene genealogies. Mol Ecol 2000, 9(10):1657-1659.

28. Templeton AR, Crandall KA, Sing CF: A cladistic analysis of phenotypic associations with haplotypes inferred from restriction endonuclease mapping and DNA sequence data. III. Cladogram estimation. Genetics 1992, 132:619-633.

29. Castelloe J, Templeton AR: Root probabilities for intraspecific gene trees under neutral coalescent theory. Mol Phylogenet Evol 1994, 3(2):102-113.

30. Huson DH: SplitsTree: analyzing and visualizing evolutionary data. Bioinformatics 1998, 14(1):68-73.

31. Morin MM, Moret BM: NETGEN: generating phylogenetic networks with diploid hybrids. Bioinformatics 2006, 22(15):1921-1923.

32. Than C, Ruths D, Nakhleh L: PhyloNet: a software package for analyzing and reconstructing reticulate evolutionary relationships. $B M C$ Bioinformatics 2008, 9:322

33. Anderson RJ, Setubal JC, Nguyen QC, Venkateswaran V, Badics T, Boros E, Alizadeh F, Goldberg AV, Shannon GE, MacCuish J, et al:: Network Flows and Matching: First DIMACS Implementation Challenge. In Network Flows and Matching Edited by: Johnson DS, McGeoch CC. Providence; 1993.

doi: $10.1186 / 1471-2105-11-268$

Cite this article as: Arenas et al., Characterization of phylogenetic networks with NetTest BMC Bioinformatics 2010, 11:268 